\title{
Induction of Ischemic Tolerance Protects the Retina From Diabetic Retinopathy
}

\author{
Diego C. Fernandez, ${ }^{\star \dagger}$ Pablo H. Sande, ${ }^{*}$ \\ Mónica S. Chianelli, ${ }^{*}$ Hernán J. Aldana Marcos, ${ }^{\dagger \neq}$ \\ and Ruth E. Rosenstein* \\ From the Laboratory of Retinal Neurochemistry and \\ Experimental Ophthalmology,* Department of Human \\ Biochemistry, School of Medicine, University of Buenos Aires, \\ CEFyBO, CONICET, Buenos Aires; the Laboratory of Histology, \\ School of Medicine, University of Morón, Buenos Aires; and the \\ Laboratory of Histology, ${ }^{\ddagger}$ School of Science, University of \\ Belgrano, Buenos Aires, Argentina
}

Diabetic retinopathy is a leading cause of acquired blindness. Available treatments are not very effective. We investigated the effect of a weekly application of retinal ischemia pulses (ischemic conditioning) on retinal damage induced by experimental diabetes. Diabetes was induced by an intraperitoneal injection of streptozotocin. Retinal ischemia was induced by increasing intraocular pressure to $120 \mathrm{mmHg}$ for 5 minutes; this maneuver started 3 days after streptozotocin injection and was weekly repeated in one eye, whereas the contralateral eye was submitted to a sham procedure. Diabetic retinopathy was evaluated in terms of i) retinal function (electroretinogram and oscillatory potentials), ii) integrity of blood-retinal barrier (by albumin-Evans blue complex leakage and astrocyte glial fibrillary acidic protein IHC), iii) optical and electron microscopy histopathologic studies, and iv) vascular endothelial growth factor levels (using Western blot analysis and IHC). Brief ischemia pulses significantly preserved electroretinogram a- and b-wave and oscillatory potentials, avoided albumin-Evans blue leakage, prevented the decrease in astrocyte glial fibrillary acidic protein levels, reduced the appearance of retinal edemas, and prevented the increase in vascular endothelial growth factor levels induced by experimental diabetes. When the application of ischemia pulses started 6 weeks after diabetes onset, retinal function was significantly preserved. These results indicate that induction of ischemic tolerance could constitute a fertile avenue for the development of new therapeutic strategies for diabetic retinopathy treatment. (Am J Pathol 2011, 178:2264-2274; DOI: 10.1016/j.ajpath.2011.01.040)
Diabetic retinopathy (DR) is a leading cause of acquired blindness. The incidence of DR is rarely detected in the first few years of diabetes, but it increases to $50 \%$ by 10 years and to $90 \%$ by 25 years of diabetes. Although DR has long been recognized as a vascular disease, it is becoming increasingly clear that retinal cells are also affected by diabetes, resulting in dysfunction and degeneration of neuronal cells. ${ }^{1}$ Vision loss occurs due to chronic hyperglycemia, vascular damage and leakage, edema, capillary basement membrane thickening, neovascularization, hemorrhage, and ischemia. ${ }^{2}$ Although the pathogenesis of DR is highly complex and not fully understood, VEGF is recognized as a major contributor to the development of DR. ${ }^{3}$ Despite the attempts to control blood glucose levels, many diabetic patients are affected by DR, which progresses to more severe forms of disease. Because available treatments are not very effective, it is imperative to develop better approaches for DR prevention and treatment. Unraveling which are the most critical mechanisms is unlikely to be achieved in studies limited to the clinically observable retinal changes in human DR. Far more detailed and invasive studies are required, preferably in a readily available animal model. ${ }^{4}$ The streptozotocin-induced diabetes in rats shows many of the retinal alterations associated with human DR. ${ }^{5-7}$ Therefore, this model could be a useful tool for studying the pathogenic mechanisms involved in DR, as well as for developing new therapeutics.

The retinal vessel tight junctions protect the vessels from leaking, but sustained hyperglycemia could damage tight junctions and the vessels could become leaky, allowing fluid or blood to seep into the retina, thus resulting in retinal swelling. ${ }^{8}$ Because of progressive dysfunction, the capillaries die prematurely, leading to ischemia, ${ }^{9,10}$ which, in turn, induces irreversible morphologic and functional changes that result in blindness. Ischemic retinopathy develops when retinal blood flow is insuffi-

Supported by grants from the Agencia Nacional de Promoción Científica y Tecnológica, The University of Buenos Aires, CONICET, Argentina, and Sigma Xi, The Scientific Research Society.

Accepted for publication January 10, 2011.

Address reprint requests to Ruth Estela Rosenstein, Ph.D., Departamento de Bioquímica Humana, Facultad de Medicina, UBA., Paraguay 2155, 5P, (1121), Buenos Aires, Argentina. E-mail: ruthr@fmed.uba.ar. 


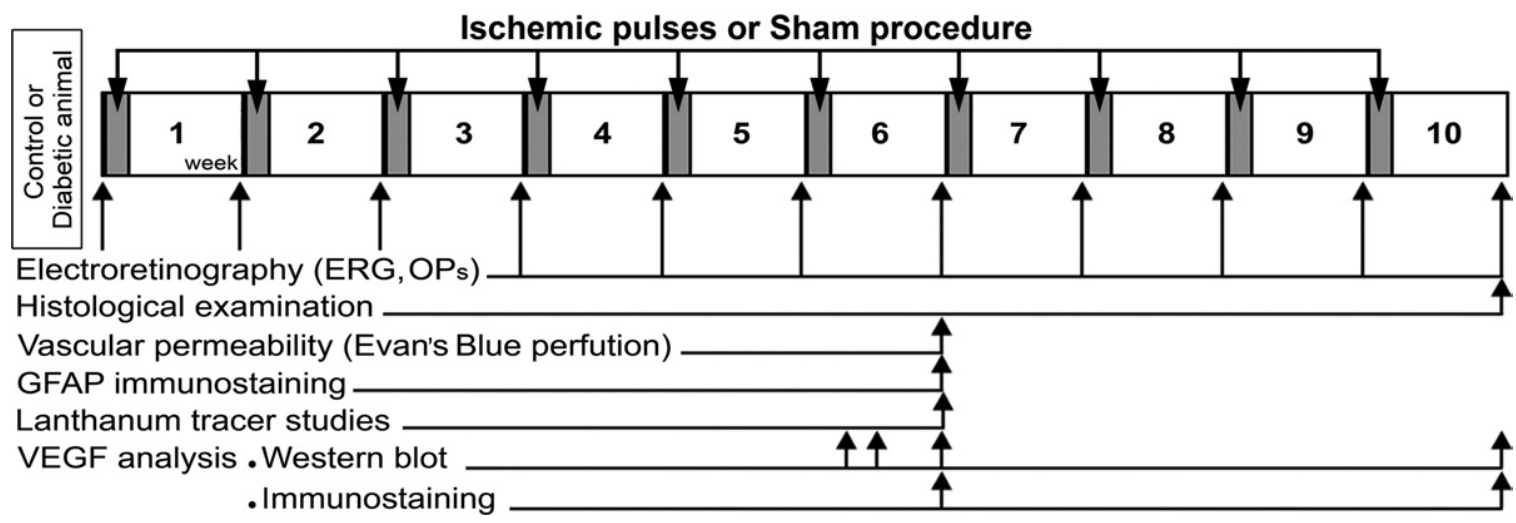

Figure 1. Experimental groups and protocols for functional and histologic studies. Three days after vehicle or streptozotocin injection, animals received a 5-minute ischemia pulse in one eye and a sham treatment in the contralateral eye. This maneuver was weekly repeated until week 10. The ERGs were weekly registered before i.p. injections and 7 days after each ischemia pulse or sham procedure application. Retinal histologic examination was performed in animals that had been diabetic for 10 weeks and were sacrificed at 7 days after the 10th ischemia pulse. Vascular permeability, GFAP immunostaining, and lanthanum tracer studies were analyzed in animals that had been diabetic for 6 weeks and were sacrificed at 7 days after the sixth ischemia pulse. The VEGF levels were analyzed using Western blot analysis and IHC at 7 days after the sixth or 10th ischemic pulse in animals that had been diabetic for 6 and 10 weeks, respectively. Moreover, VEGF levels were also assessed using Western blot analysis in animals that had been diabetic for 6 weeks and were sacrificed at 24 hours or 3 days after the sixth ischemia pulse or sham procedure.

cient to match the metabolic needs of the retina, one of the highest oxygen-consuming tissues. Although there is no effective treatment against retinal ischemic injury, it is possible to activate an endogenous protection mechanism that prevents retinal ischemic damage by ischemic preconditioning (IPC). ${ }^{11,12}$ Ischemic preconditioning requires a brief period of ischemia applied before ischemic injury, which does not produce any damage per se, and triggers yet incompletely described mechanisms that result in tolerance to the subsequent severely damaging ischemic event. ${ }^{13}$ It was shown that IPC affords the retina a greater degree of protection against ischemic damage than any known neuroprotective agent. ${ }^{11}$ Moreover, clinical studies support the effectiveness of IPC in the brain of humans with transient ischemic attacks. ${ }^{14}$

Although IPC confers robust neuroprotection in different models of ischemia, its clinical utilization is mostly limited because the onset of retinal ischemia is largely unpredictable, in contrast to the onset of reperfusion that could be more predictable. In this vein, another endogenous form of ischemic protection, in which a short series of repetitive cycles of brief ischemia/reperfusion are applied immediately at the onset of reperfusion, termed postconditioning, has been reported in several tissues. ${ }^{15-17}$ We have shown that 7-minute ischemia applied 5 minutes after the reperfusion onset induces an almost complete protection of retinas exposed to ischemic injury. ${ }^{18}$ On the basis of the highly effective protection induced by IPC and postconditioning against an acute ischemic episode, the aim of this work was to analyze the effect of brief ischemia pulses on retinal damage induced by experimental diabetes.

\section{Materials and Methods}

\section{Animals}

Male Wistar rats (300 $\pm 50 \mathrm{~g})$ were housed in a standard animal room with food and water ad libitum under controlled conditions of humidity and temperature $\left(21^{\circ} \mathrm{C} \pm 2{ }^{\circ} \mathrm{C}\right)$ and under a 12-hour light/dark cycle (lights on at 7:00 AM). All animal procedures were in strict accordance with the ARVO Statement for the Use of Animals in Ophthalmic and Vision Research. For diabetes induction, a single intraperitoneal streptozotocin injection $(60 \mathrm{mg} / \mathrm{kg}$ in $0.1 \mathrm{~mol} / \mathrm{L}$ citrate buffer, $\mathrm{pH}$ 4.5) was performed. Control rats received an equal volume of citrate buffer. Animals were examined 72 hours after injections using a glucose meter (Contour TS; Bayer, Buenos Aires, Argentina), and those with blood glucose levels $>350 \mathrm{mg} / \mathrm{dL}$ were considered diabetic. For the assessment of body weight and blood glucose levels, a group of diabetic animals with intact eyes was included. Rats were monitored weekly, and the weight and plasma glucose levels were determined.

\section{Ischemic Conditioning Protocol}

Animals were anesthetized with ketamine hydrochloride $(150 \mathrm{mg} / \mathrm{kg})$ and xylazine hydrochloride $(2 \mathrm{mg} / \mathrm{kg})$ administered intraperitoneally. After topical instillation of proparacaine, the anterior chamber was cannulated with a 30-gauge needle connected to a pressurized bottle filled with sterile saline solution. Rats were submitted to different treatments depicted in Figure 1. Three days after vehicle or streptozotocin injection, retinal ischemia was induced by increasing intraocular pressure to $120 \mathrm{~mm}$ $\mathrm{Hg}$ for exactly 5 minutes, as previously described. ${ }^{18}$ With this maneuver, complete ocular ischemia was produced, characterized by the loss of the electroretinogram (ERG) b-wave and the cessation of flow in retinal vessels, determined by funduscopic examination. The contralateral eye was cannulated without raising intraocular pressure (sham procedure). During ischemia pulses, animals were kept normothermic with heated blankets. This maneuver was repeated weekly until week 10 (Figure 1). In a group of nondiabetic animals, only one eye was subjected weekly to 5-minute ischemia, whereas the contralateral eye remained intact. In another set of experiments, ischemia pulses started after 6 weeks of diabetes induction and continued until week 12 . 


\section{Electroretinography}

Electroretinographic activity was assessed before and weekly after diabetes induction, as previously described. ${ }^{18}$ Briefly, after 6 hours of dark adaptation, rats were anesthetized under dim red illumination. Phenylephrine hydrochloride and tropicamide were used to dilate the pupils, and the cornea was intermittently irrigated with balanced salt solution to maintain the baseline recording and to prevent keratopathy. Rats were placed facing the stimulus at a distance of $20 \mathrm{~cm}$. All recordings were completed within 20 minutes and animals were kept warm during and after the procedure. A reference electrode was placed through the ear, a grounding electrode was attached to the tail, and a gold electrode was placed in contact with the central cornea. A $15-W$ red light was used to enable accurate electrode placement. This maneuver did not significantly affect dark adaptation and was switched off during the electrophysiologic recordings. Electroretinograms were recorded from both eyes simultaneously, and 10 responses to flashes of unattenuated white light $(5 \mathrm{~ms}, 0.2 \mathrm{~Hz}$ ) from a photic stimulator (light-emitting diodes) set at maximum brightness (6 $\mathrm{cd} \mathrm{s} / \mathrm{m}^{2}$ without filter) were amplified, filtered $(1.5-\mathrm{Hz}$ lowpass filter, 1000 high-pass filter, notch activated), and averaged (Akonic BIO-PC, Buenos Aires, Argentina). The awave was measured as the difference in amplitude between the recording at onset and the trough of the negative deflection, and the b-wave amplitude was measured from the trough of the a-wave to the peak of the b-wave. Runs were repeated 3 times with 5-minute intervals to confirm consistency. Mean values from each eye were averaged, and the resultant mean value was used to compute the group means \pm SEMs of a- and b-wave amplitude. The mean peak latencies and peak-to-peak amplitudes of the responses from each group of rats were compared.

Oscillatory potentials (OPs) were assessed using the same photic stimulator with a $0.2-\mathrm{Hz}$ frequency and filters of high $(300 \mathrm{~Hz})$ or low $(100 \mathrm{~Hz})$ frequency. The amplitudes of the OPs were estimated by measuring the heights from the baseline drawn between the troughs of successive wavelets to their peaks. The sum of three OPs was used for statistical analysis. The ERGs were registered before ischemia pulses.

\section{Histologic Examination}

Rats were anesthetized and intracardially perfused with $150 \mathrm{~mL}$ of saline solution followed by $300 \mathrm{~mL}$ of a fixative solution containing $4 \%$ formaldehyde in $0.1 \mathrm{~mol} / \mathrm{L}$ phosphate buffer ( $\mathrm{pH} 7.2)$. Then, eyes were carefully enucleated, immersed for 24 hours in the same fixative, and embedded in paraffin. Eyes were sectioned ( $5 \mu \mathrm{m}$ ) along the vertical meridian through the optic nerve head. Sections were stained with hematoxylin-eosin and were analyzed by masked observers. Microscopic images were digitally captured with a microscope (6-V halogen lamp, $20 \mathrm{~W}$, equipped with a stabilized light source, Nikon Eclipse E400; Nikon, Abingdon, VA) attached to a digital camera (Coolpix s10; Nikon). For morphometric analysis, digitalized images captured were processed using Scion Image for Windows software (Scion Corporation, Freder- ick, MD). The average total retina and retinal layer thicknesses (in micrometers) for each eye were measured, and the number of cells in the ganglion cell layer (GCL) per $200 \mu \mathrm{m}$ was determined in the same topographic region of the retina. Measurements $(\times 200)$ were obtained $1 \mathrm{~mm}$ dorsally and ventrally from the optic disc. For each eye, results obtained from four separate sections were averaged, and the mean of five eyes was recorded as the representative value for each group.

\section{Immunohistochemical Studies}

Antigen retrieval was performed by heating sections at $90^{\circ} \mathrm{C}$ for 30 minutes in citrate buffer $(\mathrm{pH} \mathrm{6.3)}$ and then preincubated with $2 \%$ normal horse serum, $0.1 \%$ bovine serum albumin, and $0.4 \%$ Triton X-100 in $0.01 \mathrm{~mol} / \mathrm{L}$ PBS for 1 hour. For vascular endothelial growth factor (VEGF) immunodetection, paraffin sections were treated with $0.3 \%$ $\mathrm{H}_{2} \mathrm{O}_{2}$ in PBS for 20 minutes (for blocking endogenous peroxidase activity) and incubated overnight at $4^{\circ} \mathrm{C}$ with a rabbit polyclonal anti-VEGF antibody (1:800; Calbiochem, La Jolla, CA). An immunohistochemical detection was performed using the LSAB2 System-HRP (Dako, Carpinteria, California), based on biotin-streptavidin-peroxidase, and visualized using 3,3'-diaminobenzidine as chromogen.

For neuronal nuclear protein (NeuN) immunodetection, paraffin sections were incubated overnight at $4^{\circ} \mathrm{C}$ with a mouse monoclonal anti-NeuN antibody (1:200; Millipore, Bilerica, MA), and then an anti-mouse secondary antibody conjugated to Alexa Fluor 568 (1:500; Molecular Probes, Eugene, OR) was used. After immunostaining, nuclei were stained with the fluorescent dye DAPI. Measurements of cell number were performed as described above.

For immunodetection of glial cells, rats were perfused as previously described. Perfusion pressure was kept low to minimize damage to vessels. The posterior eye cups were immersed in an ice-cold fixative for 4 hours. Retinas were carefully detached and flat-mounted with the vitreous side up in Superfrost microscope slides (Erie Scientific Company, Portsmouth, $\mathrm{NH}$ ). Whole-mount retinas were incubated overnight at $4^{\circ} \mathrm{C}$ with a mouse monoclonal anti-glial fibrillary acidic protein (GFAP) antibody conjugated to Cy3 (1:400; Sigma Chemical Co., St. Louis, MO). Specimens were mounted with antifade medium (Vectashield; Vector Laboratories, Burlingame, CA) and viewed with a fluorescence microscope (BX-50; Olympus, Tokyo, Japan) mounted with a video camera (3CCD; Sony, Tokyo, Japan) attached to a computer running image analysis software (Optimus; Media Cybernetics, Silver Spring, MD). In all cases, comparative digital images from different samples were obtained using identical exposure time, brightness, and contrast settings. The retina was divided into four quadrants, and images from the central (peripapillary region) and peripheral area were obtained. The area occupied by GFAP-positive astrocytes was measured in a square area corresponding to $0.125 \mathrm{~mm}^{2}$ and expressed as a percentage of the total area. For each group, results obtained from four separate quadrants were averaged, and the mean of four eyes was recorded as the representative value. Regularly, some sections were treated without the primary antibodies to confirm specificity. 
Table 1. Average Body Weight and Blood Glucose Concentration Assessed at Different Time Points

\begin{tabular}{|c|c|c|c|c|c|c|}
\hline \multirow[b]{2}{*}{$\begin{array}{l}\text { Time after vehicle or } \\
\text { streptozotocin } \\
\text { injection }\end{array}$} & \multicolumn{3}{|c|}{ Average body weight, $g$} & \multicolumn{3}{|c|}{ Average blood glucose concentration, mg/dL } \\
\hline & Control & $\begin{array}{l}\text { Diabetes without } \\
\text { ischemia pulses }\end{array}$ & $\begin{array}{l}\text { Diabetes with } \\
\text { ischemia } \\
\text { pulses }\end{array}$ & Control & $\begin{array}{l}\text { Diabetes without } \\
\text { ischemia pulses }\end{array}$ & $\begin{array}{c}\text { Diabetes with } \\
\text { ischemia } \\
\text { pulses }\end{array}$ \\
\hline 3 days & $315.6 \pm 9.4$ & $337.6 \pm 10.0$ & $333.7 \pm 8.4$ & $109.4 \pm 6.4$ & $484.7 \pm 16.5^{\star}$ & $476.0 \pm 15.4^{*}$ \\
\hline 3 weeks & $404.3 \pm 5.1$ & $320.0 \pm 7.9^{*}$ & $305.4 \pm 5.2^{*}$ & $110.3 \pm 4.3$ & $498.1 \pm 12.7^{\star}$ & $502.3 \pm 12.9^{*}$ \\
\hline 6 weeks & $432.6 \pm 4.7$ & $317.2 \pm 11.1^{*}$ & $306.7 \pm 12.2^{*}$ & $105.9 \pm 5.3$ & $519.8 \pm 16.5^{*}$ & $528.3 \pm 16.2^{*}$ \\
\hline 10 weeks & $461.2 \pm 6.1$ & $307.8 \pm 19.2^{*}$ & $300.6 \pm 18.5^{\star}$ & $117.5 \pm 4.3$ & $583.4 \pm 9.8^{\star \dagger}$ & $596.3 \pm 8.3^{* \dagger}$ \\
\hline
\end{tabular}

The injection of streptozotocin induced a significant decrease in body weight and an increase in blood glucose levels. Ischemia pulses in streptozotocininjected rats did not change these parameters. Data are given as mean \pm SEM $(n=10$ animals per group). No significant differences in the average body weight were observed in diabetic animals during the study.

${ }^{*} P<0.01$ versus aged-matched control animals, by Tukey's test.

${ }^{\dagger} P<0.01$ versus 3 days after streptozotocin injection, by Dunnett's test.

\section{Vascular Permeability}

Vascular permeability was analyzed by measuring albumin-Evans blue complex leakage from retinal vessels as previously described. ${ }^{19}$ Briefly, animals were anesthetized and injected intracardially with a solution of Evans blue (2\% wt/vol dissolved in PBS). Immediately after injection, animals turned visibly blue, confirming the dye uptake and distribution. After 40 minutes, animals were sacrificed and flat-mounted retinas were obtained as described above. Microphotographs were obtained using identical exposure time, brightness, and contrast settings. For each group, results were qualitatively analyzed by comparing four eyes for group.

\section{Lanthanum Tracer Studies}

After anesthesia and thoracotomy, animals were perfused through the left ventricle with three perfusates, according to DePace, ${ }^{20}$ with minimal modifications. The initial perfusate was 100 to $150 \mathrm{~mL}$ of $1 \% \mathrm{NaNO}_{3}$ in
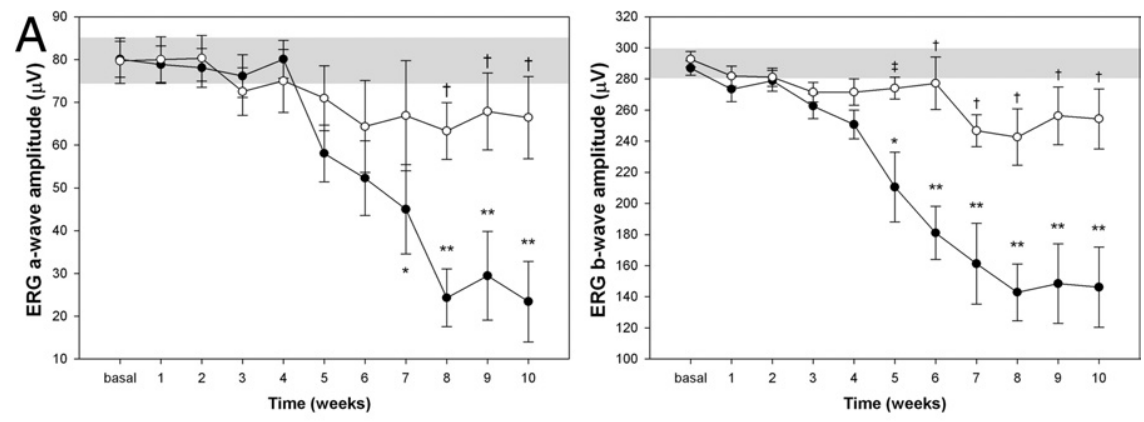

B

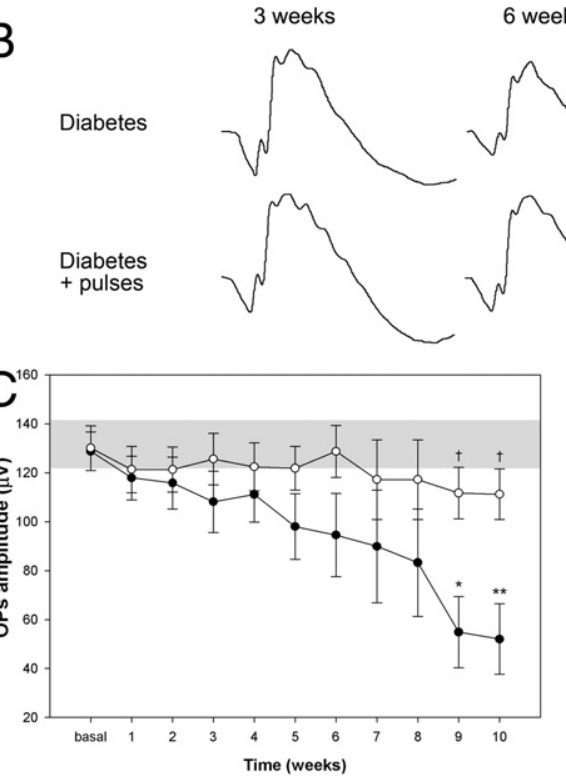

10 weeks

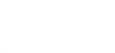

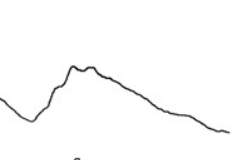
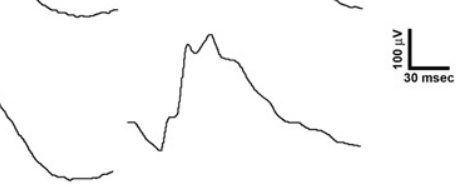

$D_{3 \text { weeks }}$

6 weeks

10 weeks

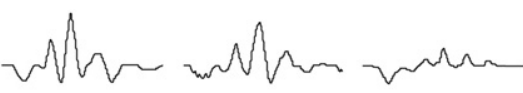
Diabetes

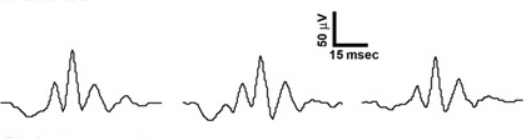

Diabetes + pulses
Figure 2. Temporal course of ERG protection in duced by the application of brief pulses of ischemia against diabetic damage. The ERGs (A) and OPs $(\mathbf{C})$ were assessed weekly after the induction of diabetes. After 7 and 5 weeks, diabetes (black circles) induced a significant decrease in ERG aand b-wave amplitude, respectively, compared with age-matched control animals (gray bar). In diabetic eyes submitted to ischemia pulses (white circles), a significant prevention of these alterations was observed. B: Representative scotopic ERG traces from both eyes (sham or ischemia pulses) of diabetic animals assessed 3, 6, and 10 weeks after induction of diabetes. C: A significant decrease in the sum of OP amplitude was observed after 9 weeks of diabetes onset, whereas ischemia pulses prevented this effect. D: Representative OP traces from both eyes (sham and ischemia pulses) of diabetic animals assessed 3, 6, and 10 weeks after the induction of diabetes. No changes in OP or ERG a- and b-wave latencies were observed among groups. Data are the mean $\pm \operatorname{SEM}(n=12$ eyes per group); ${ }^{*} P<0.05$, ${ }^{* * *} P<0.01$ versus age-matched control; ${ }^{\ddagger} P<0.05,{ }^{\dagger} P<0.01$ versus diabetic sham-treated eyes, by Tukey's test. 

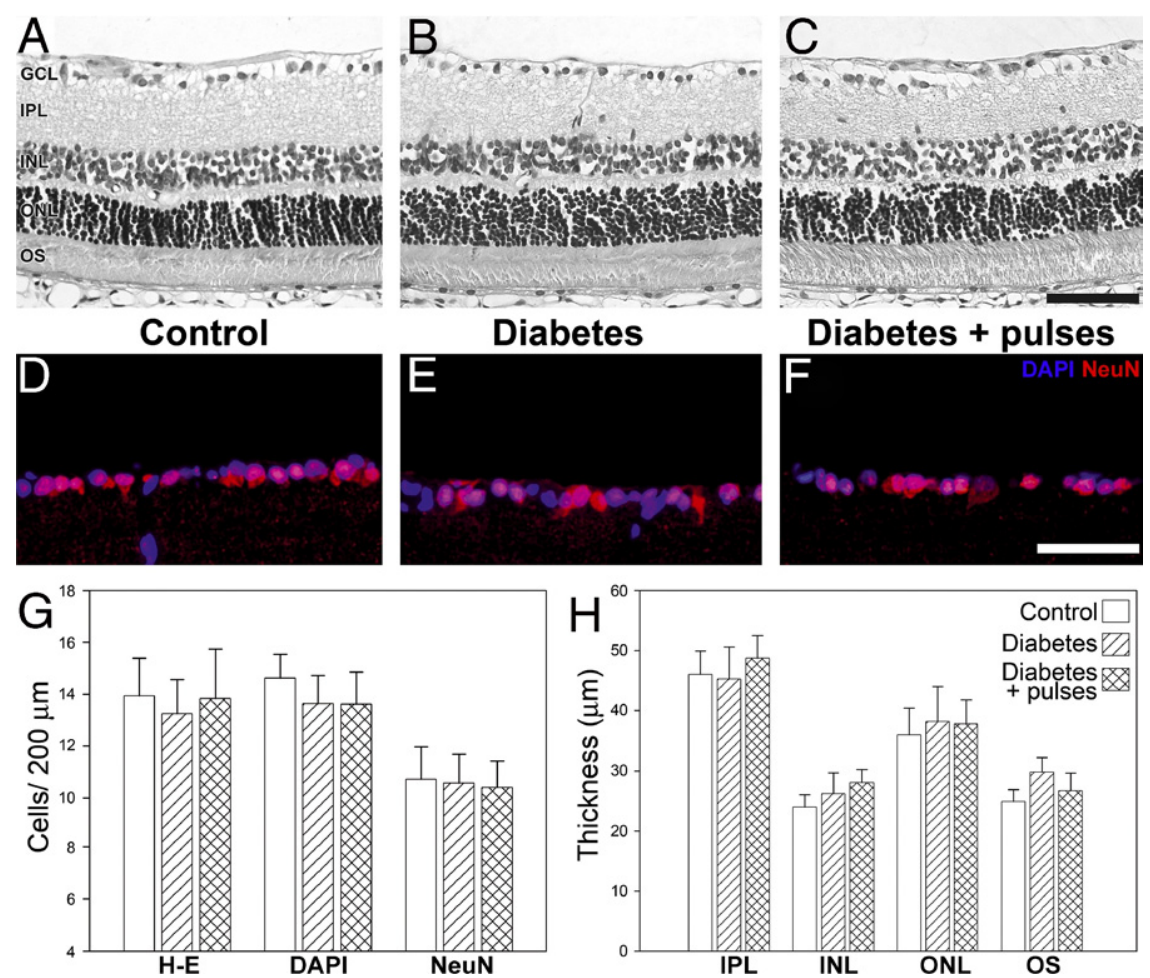

Figure 3. Retinal histologic examination after 10 weeks of diabetes. Representative photomicrographs of retinal sections from a control eye (A) and a diabetic eye without (B) or with (C) pulses of ischemia. No evident alterations in retinal morphologic features were observed. Im munohistochemical detection of NeuN-positive neurons in the GCL from a control eye (D) and a diabetic eye without (E) or with (F) ischemia pulses. A strong NeuN immunostaining was confined to ganglion cells in the GCL (red). Cell nuclei were counterstained with DAPI (blue) Cell count in the GCL evaluated by hematoxylineosin staining, DAPI staining, and NeuN immunostaining $(\mathbf{G})$. No differences in the number of cells were observed by these methods. Retinal layer thickness assessed in different groups after 10 weeks of diabetes $(\mathbf{H})$. Whole retina thickness was $137.5 \pm 12.3 \mu \mathrm{m}, 158.2 \pm 10.8 \mu \mathrm{m}$, and $152.0 \pm 19.2 \mu \mathrm{m}$ in control, diabetic, and diabetic with ischemia pulses groups, respectively. These values did not differ among groups. Scale bar: $50 \mu \mathrm{m}$ (upper); $100 \mu \mathrm{m}$ (middle). Data are the mean \pm SEM ( $n=5$ eyes per group). IPL, inner plexiform layer; INL, inner nuclear layer; ONL, outer nuclear layer; OS, outer segments of photoreceptors
PBS, immediately followed by a 5-minute infusion with 300 to $350 \mathrm{~mL}$ of ionic lanthanum solution containing $40 \mathrm{mmol} / \mathrm{L} \mathrm{La}\left(\mathrm{NO}_{3}\right)_{3} .6 \mathrm{H}_{2} \mathrm{O}, 80 \mathrm{mmol} / \mathrm{L} \mathrm{NaCl}, 3.5$ $\mathrm{mmol} / \mathrm{L} \mathrm{KCl}, 1 \mathrm{mmol} / \mathrm{L} \mathrm{CaCl}, 1 \mathrm{mmol} / \mathrm{L} \mathrm{MgCl}_{2}$, and 1 $\mathrm{mmol} / \mathrm{L}$ glucose at $\mathrm{pH}$ 7.4. Lanthanum nitrate solution was followed by an additional 10-minute perfusion with 300 to $350 \mathrm{~mL}$ of $2 \%$ glutaraldehyde and $4 \%$ paraformaldehyde prepared in a sulfate-salt solution containing $43 \mathrm{mmol} / \mathrm{L} \mathrm{NaLa}\left(\mathrm{NO}_{3}\right) 3.6 \mathrm{H}_{2} \mathrm{O}, 80 \mathrm{mmol} / \mathrm{L} \mathrm{NaCl}, 3.5$ $\mathrm{mmol} / \mathrm{L} \mathrm{KCl}, 1.0 \mathrm{mmol} / \mathrm{L} \mathrm{CaCl} \mathrm{SO}_{4}, 16 \mathrm{mmol} / \mathrm{L} \mathrm{NaHCO}, 10$ $\mathrm{mmol} / \mathrm{L}$ sodium acetate, $3.5 \mathrm{mmol} / \mathrm{L} \mathrm{KCl}, 1.0 \mathrm{mmol} / \mathrm{L} \mathrm{CaCl}_{2}$, $1.0 \mathrm{mmol} / \mathrm{L} \mathrm{MgCl}_{2}, 1.0 \mathrm{mmol} / \mathrm{L}$ glucose, $1.6 \mathrm{mmol} / \mathrm{L}$ $\mathrm{Na}_{2} \mathrm{HPO}_{4}, 0.4 \mathrm{mmol} / \mathrm{L} \mathrm{NaH} \mathrm{PO}_{4}$, and $33 \mathrm{mmol} / \mathrm{L}$ sucrose at $\mathrm{pH}$ 7.4. After perfusion, retinas were dissected out, and after several washings, tissue blocks were postfixed in $2 \%$ aqueous osmium tetroxide for 1 hour. Dehydration was accomplished by gradual ethanol series and tissue samples were embedded in epoxy resin. Semithin sections were stained with toluidine blue and ultrathin sections were stained with uranyl acetate and lead citrate. Afterward, sections were viewed and photographed using a transmission electron microscope (Zeiss EM 10 C; Zeiss, Oberkochen, Germany). For each group, results were qualitatively analyzed by comparing four eyes for group.

\section{Western Blotting}

Animals were sacrificed at 6 or 10 weeks after diabetes induction at 7 days after the sixth and 10th ischemic pulses, respectively. Moreover, VEGF levels were also assessed in animals that had been diabetic for 6 weeks and were sacrificed at 24 hours or 3 days after the sixth ischemia pulse (Figure 1). Retinas (one per condition) were homogenized in $150 \mu \mathrm{L}$ of a buffer containing 10 $\mathrm{mmol} / \mathrm{L}$ HEPES, $1 \mathrm{mmol} / \mathrm{L}$ EDTA, $1 \mathrm{mmol} / \mathrm{L}$ EGTA, 10 $\mathrm{mmol} / \mathrm{L} \mathrm{KCl}$, Triton 0.5\% ( $\mathrm{vol} / \mathrm{vol}), \mathrm{pH} 7.9$, supplemented with a cocktail of protease inhibitors (Sigma Chemical Co.). After 15 minutes at $4^{\circ} \mathrm{C}$, homogenates were gently vortexed for 15 seconds and centrifuged at $3000 \times g$ for 10 minutes. Supernatants were used to determine protein concentration. Proteins (50 $\mu \mathrm{g}$ per sample) were separated in SDS, $12 \%$ polyacrylamide gel electrophoresis. After electrophoresis, proteins were transferred to polyvinylidene difluoride membranes for 60 minutes at $15 \mathrm{~V}$ in a Bio-Rad Trans-Blot SD system (Bio-Rad Laboratories, Hercules, CA). Membranes were blocked in 5\% nonfat dry milk in Tris-buffered saline $(\mathrm{pH} 7.4)$ containing $0.1 \%$ Tween 20 for 60 minutes at room temperature and then incubated overnight at $4{ }^{\circ} \mathrm{C}$ with a rabbit anti-VEGF antibody (1:1000). Membranes were washed and then incubated for 1 hour with a horseradish peroxidase-conjugated secondary antibody (1:2000). Immunoblots were visualized by enhanced chemiluminescence Western blotting detection reagents (Amersham Biosciences). Autoradiographic signals were quantified by densitometry using ImageQuant software and adjusted by the density of $\beta$-actin.

Protein content was determined by the method of Lowry et $a^{21}{ }^{21}$ using bovine serum albumin as the standard.

\section{Statistical Analysis}

Statistical analysis was made by a two-way analysis of variance followed by Tukey's test. 

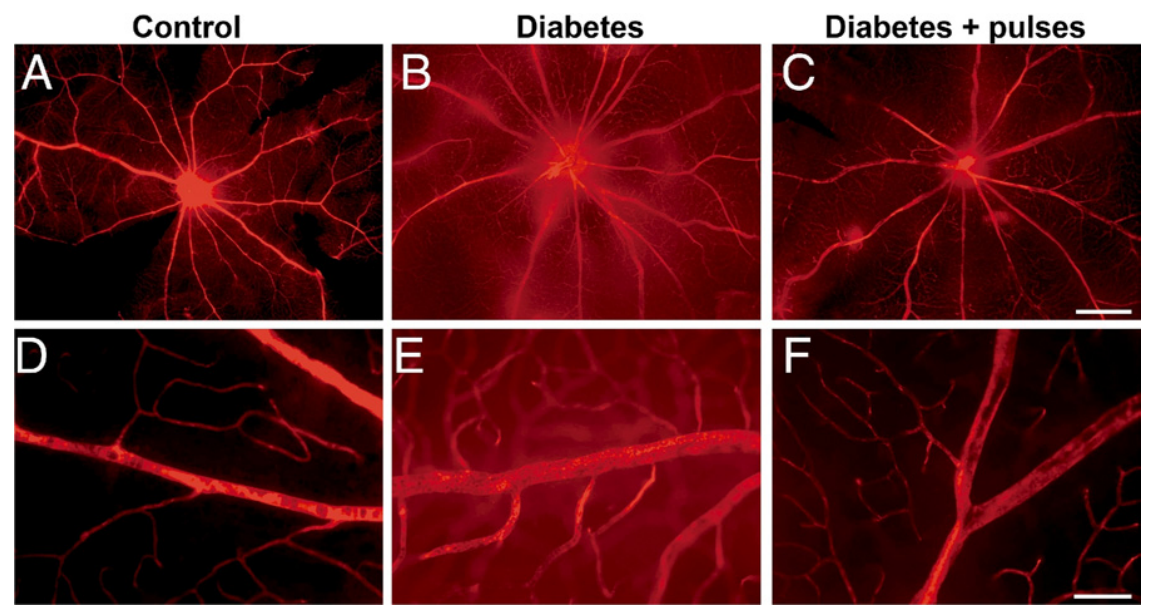

Figure 4. Visualization of retinal blood vessels leakage after intravascular perfusion with Evans blue dye. In control retinas ( $\mathbf{A}$, detail in $\mathbf{D})$, the superficial (in focus) and deeper vasculature was sharply outlined by the dye, showing very low levels of background fluorescence. At 6 weeks of diabetes, in retinas from eyes submitted to a sham procedure (B, detail in $\mathbf{E}$ ), focal sites of leakage were noted, with the dye diffusely distributed through the retinal parenchyma. The severity of the vascular permeability was markedly reduced in diabetic eyes submitted to ischemia pulses ( $\mathbf{C}$, detail in $\mathbf{F}$ ), with minor focal leakage. Scale bar: $500 \mu \mathrm{m}(\mathbf{A}-\mathbf{C}) ; 50$ $\mu \mathrm{m}(\mathbf{D}-\mathbf{F})$. Shown are photographs representative of four eyes per group.

\section{Results}

Table 1 summarizes the average weight and blood glucose levels after the injection of vehicle or streptozotocin. At 3, 6, and 10 weeks after injections, a significant weight loss of streptozotocin-treated rats compared with vehicleinjected rats was observed. Blood glucose levels were significantly increased at these time points in streptozotocin-injected rats. The weekly application of 5-minute ischemia pulses did not change these parameters compared with diabetic animals without ischemia pulses.

Figure 2A depicts the temporal course of the electroretinographic activity in control and diabetic animals with- out or with ischemia pulses. Representative ERG waveforms registered at different time points of diabetes are shown in Figure 2B. No ERG changes were noticed in control rats during the entire period of analysis. In diabetic eyes without ischemia pulses, a progressive decrease in the scotopic ERG a- and b-wave amplitude was observed, reaching significance at 7 and 5 weeks after injection of streptozotocin, respectively. In eyes submitted to ischemia pulses, the diabetes-induced decrease in ERG a- and b-wave amplitude was significantly prevented. The sum of OP amplitudes significantly decreased after 9 weeks of diabetes, compared with vehi-
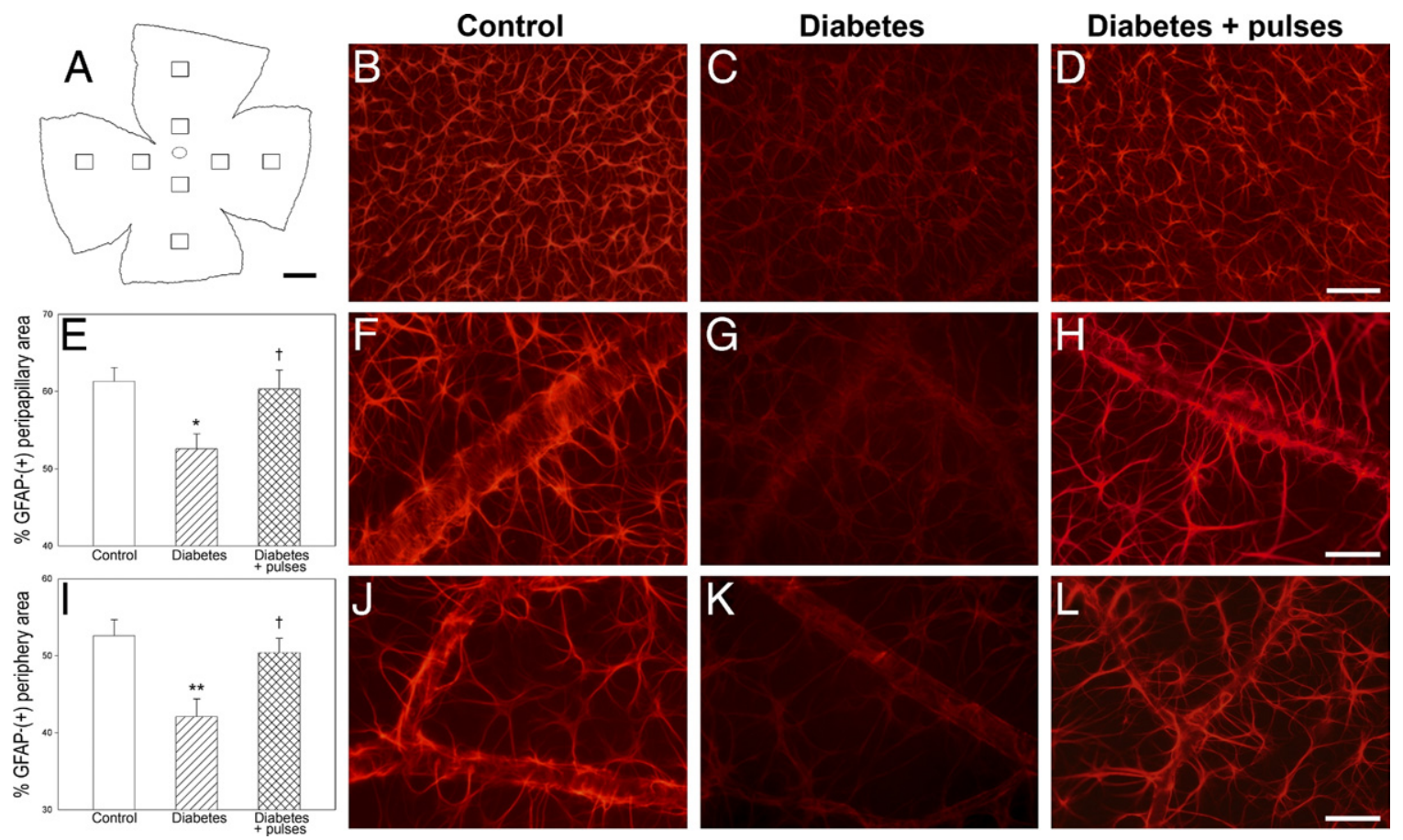

Figure 5. Assessment of astrocyte GFAP immunoreactivity after 6 weeks of diabetes induction. A: Schematic diagram of a flat-mounted retina showing all of the regions analyzed. In control retinas $(\mathbf{B})$, astrocytes were intensely immunoreactive for GFAP in the central (F) and peripheral regions ( $\mathbf{J})$. Note that blood vessels and astrocytes end processes were clearly visible. The intensity of GFAP immunoreactivity was significantly reduced in diabetic eyes submitted to a sham procedure $(\mathbf{C})$, in both the peripapillary $(\mathbf{E}$ and $\mathbf{G})$ and peripheral regions (I and $\mathbf{K})$. In eyes from diabetic animals that received a weekly application of brief pulses of ischemia, a prevention of the decrease in GFAP immunoreactivity induced by diabetes was observed (D). This prevention was evident in all of the quadrants analyzed, both in central ( $\mathbf{E}$ and $\mathbf{H})$ and peripheral (I and $\mathbf{L})$ regions. Data are the mean \pm SEM $\left(n=4\right.$ eyes per group); ${ }^{*} P<0.05,{ }^{* * *} P<0.01$ versus age-matched controls; ${ }^{\dagger} P<0.05$ versus diabetic sham-treated eyes, by Tukey's test. Scale bar: $100 \mu \mathrm{m}(\mathbf{B}-\mathbf{D}) ; 50 \mu \mathrm{m}(\mathbf{F}-\mathbf{H}, \mathbf{J}-\mathbf{L})$. 
cle-injected animals, whereas ischemia pulses prevented the effect of diabetes (Figure 2C). No significant differences in the ERG between control eyes and eyes submitted to ischemia pulses were observed along the study (data not shown).

No apparent morphologic differences were observed among groups. A morphometric analysis of retinal sections performed 10 weeks after streptozotocin injection revealed no differences in the total retina and retinal layers thickness, as well as in the number of cells in the GCL, as shown in Figure 3. In addition, NeuN-immunopositive neuron density in the GCL did not differ between retinas from diabetic animals submitted to a sham procedure or ischemia pulses (Figure 3).

The blood retinal barrier (BRB) integrity was analyzed in flat-mounted retinas by the albumin-Evans blue-complex leakage method, 6 weeks after diabetes induction (Figure 4). In nondiabetic animals, the dye was exclusively observed within the vessel lumen of the complete superficial and deeper retinal vasculature, with very low background fluorescence levels. In diabetic animals submitted to a sham procedure, a generalized leakiness and focal dye leakage from the optic disc region and larger vessels was observed. In eyes from diabetic animals with ischemia pulses, a remarkable reduction of vascular permeability was evident, and only some focal leakages were observed in larger vessels.

To investigate astrocytes alterations, GFAP immunoreactivity was analyzed in flat-mounted retinas. In control retinas, an intense GFAP immunoreactivity was observed in star-shaped astrocytes forming a single cell layer adjacent to the inner limiting membrane (Figure 5). At 6 weeks of diabetes, a clear reduction in GFAP immunoreactivity in all of the analyzed regions was observed. In eyes with ischemia pulses, GFAP immunoreactivity was similar to that observed in nondiabetic eyes (Figure 5).

To analyze the BRB at the ultrastructural level, a lanthanum-tracing analysis was performed. The general morphologic features of the blood vessels were similar in different groups. No evident changes in pericyte structure or in the basement membrane thickness were observed (Figure 6). In control retinas, lanthanum deposits were found in blood vessel lumen, and no evidence of tight junction breakdown was observed (Figure 6A). After 6 weeks of diabetes, only a few small vessels with altered integrity of BRB were found (Figure 6B). At higher magnification, the tracer was found filling almost the entire length of intercellular junctions of endothelial cells reaching the basal lamina (Figure 6D). Frequent edemas were observed at the level of endothelial junctions (Figure 6, B and $E$ ). In comparison with control retinas, endothelial cells showed a high number of micropinocytotic pits at the luminal E-face and intraendothelial cell vesicles $(0.60$ to $1.10-\mu \mathrm{m}$ diameter) filled with lanthanum deposits (Figure 6F). All these alterations were observed in both the inner and outer vascular plexus. In diabetic eyes submitted to ischemia pulses, no evidence of tight junction breakdown or edema formation were noted (Figure 6C), but lanthanum-filled intraendothelial cell vesicles were observed (Figure 6G).

The protein levels and cellular localization of VEGF were examined using Western blot analysis and $\mathrm{IHC}$, respectively (Figure 7). After 6 weeks of diabetes onset, VEGF levels increased approximately twofold compared with control retinas. Ischemia pulses partially but significantly prevented the diabetes-induced increase in VEGF levels at 3 and 7 days (but not 24 hours) after the sixth ischemia pulse (Figure 7B). These results were further con-

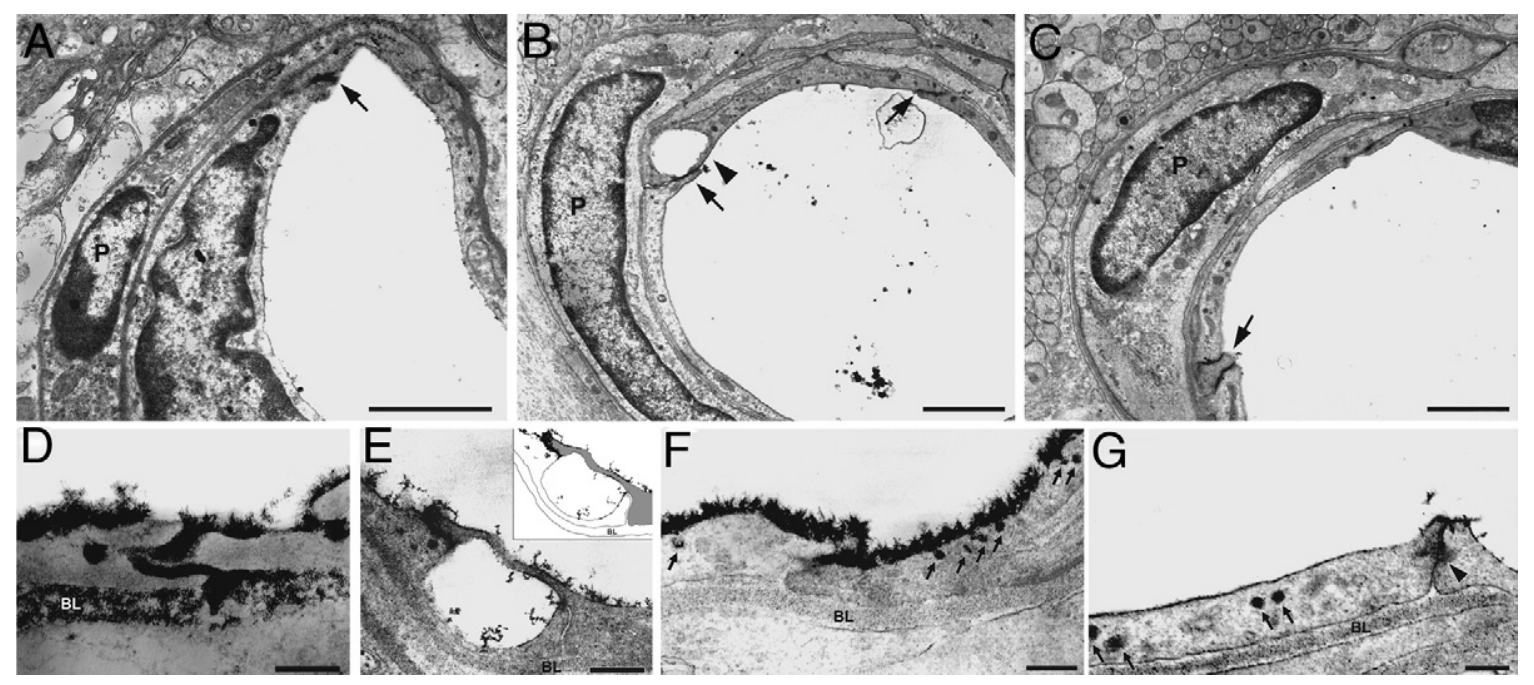

Figure 6. Ultrastructural examination of the inner BRB integrity assessed by a lanthanum tracing method at 6 weeks of diabetes. Transverse sections of vessels from a control eye (A), a diabetic eye submitted to a sham treatment (B), or a ischemic conditioning (C). Note that vessels were surrounded by a continuous basal lamina (BL) with an associated pericyte (P) in all groups. In diabetic retinas, lanthanum was localized on the luminal surface of endothelial cells and filling intercellular spaces (B, arrows). Frequent edemas were observed (arrowhead). At higher magnification, only a few junctions with the presence of lanthanum filling the intercellular space and reaching the basal lamina were found (D). In some cases, edemas were observed at the junction level (E). Note the integrity of both endothelial cells (see the diagram in the inset) surrounding the edema and the complete absence of lanthanum deposits at the basal lamina. The tracer was also observed in a high number of micropinocytotic pits and cytoplasmic vesicles at the luminal E-face of endothelial cells (arrows, F). The application of ischemia pulses prevented the occurrence of edemas but intraendothelial cell vesicles filled with lanthanum deposits persisted (arrows, G). In all of the cases evaluated, the tracer was blocked half way down the intercellular space by tight junctions between endothelial cells $(\mathbf{G}$, arrowhead). Scale bar: $1.5 \mu \mathrm{m}(\mathbf{A}-\mathbf{C})$; $0.6 \mu \mathrm{m}(\mathbf{E})$; and $0.3 \mu \mathrm{m}(\mathbf{D}, \mathbf{F}$, and $\mathbf{G})$. Shown are microphotographs representative of four eyes per group. 

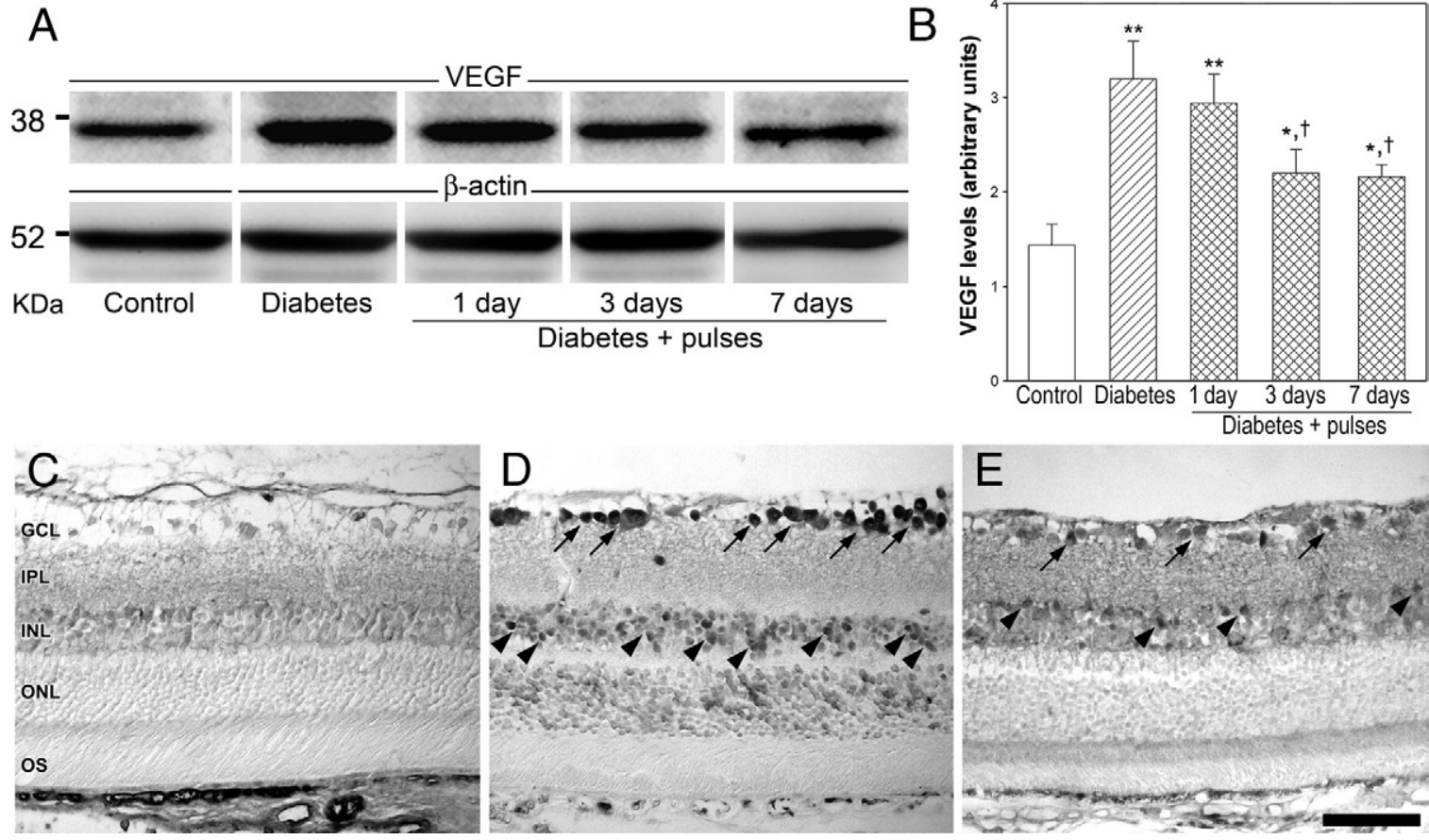

Figure 7. Retinal VEGF levels assessed using Western blot analysis and IHC. A: Representative Western blot analysis performed 6 weeks after the injection of streptozotocin at 24 hours and 3 and 7 days after the sixth ischemia pulse. B: Densitometric analysis of all samples. VEGF levels significantly increased in retinas from diabetic eyes submitted to a sham procedure compared with age-matched controls. This increase was partially prevented at 3 and 7 days (but not 24 hours) after the sixth ischemia pulse. Lower panel: Localization of VEGF protein in retinal sections assessed 6 weeks after the injection of vehicle or streptozotocin. In control retinas (C), a weak immunoreactivity was diffusely observed throughout the inner retina. In retinas from diabetic rats submitted to a sham procedure, intense immunoreactive perikarya in GCL cells (D, arrows) and in some cells in the inner nuclear layer (INL) (arrowheads) were observed. A slight immunoreactivity for VEGF was also observed in the outer retina. The weakly application of ischemia pulses prevented this effect, and only moderate VEGF immunoreactivity was found in the inner retina $(\mathbf{E})$. No immunoreactivity was detected in the staining without primary antibody (data not shown). Scale bar $=$ $50 \mu \mathrm{m}(\mathbf{C}-\mathbf{E})$. IPL, inner plexiform layer; ONL, outer nuclear layer; OS, outer segments of photoreceptors. Data are the mean \pm SEM $\left(n=4\right.$ eyes per group); ${ }^{*} P$ $<0.05$, ${ }^{* * *} P<0.01$ versus age-matched controls; ${ }^{\dagger} P<0.01$ versus diabetic sham-treated eyes, by Tukey's test.

firmed by IHC. In nondiabetic animals, a weak VEGF immunoreactivity was observed in the inner retina (Figure $7 \mathrm{C}$ ), whereas after 6 weeks of diabetes, an intense immunolabeling was observed in perikarya of neurons in the GCL and some cells in the inner and outer nuclear layer (Figure 7D). Ischemia pulses reduced VEGF immunoreactivity to levels comparable with those found in nondiabetic eyes (Figure $7 \mathrm{E})$. A similar profile was observed in retinas from streptozotocin-injected rats at 10 weeks after injection (data not shown).

To analyze the therapeutic effect of ischemia pulses, the application of pulses started 6 weeks after the injection of streptozotocin (Figure 8A). In diabetic animals, a continuous decline in the retinal function was observed. However, weekly ischemia pulses provoked a marked reversion of the diabetes-induced decrease in the ERG a- and b-wave amplitude (Figure 8, C and D). Similar results were observed for the OP amplitude (Figure 8E).

\section{Discussion}

For the first time, to our knowledge, the present results demonstrate that a weekly application of 5-minute retinal ischemia pulses, which showed no effect per se, prevented retinal alterations induced by experimental diabetes, probably through a VEGF-dependent mechanism. A single in- jection of streptozotocin in rats is a well-validated model for DR. Indeed, streptozotocin injection induced a sustained hyperglycemia, decreased body weight, and provoked retinal alterations, which are compatible with human DR. Notably, the protection induced by ischemia pulses was independent from the glycemic profile, as shown by the fact that ischemia pulses did not affect hyperglycemia and the weight loss induced by streptozotocin.

Early changes in several components of dark-adapted ERG in streptozotocin-treated rats have been previously described. ${ }^{22}$ In agreement, a significant and progressive ERG dysfunction was observed in eyes from rats injected with streptozotocin. The weekly application of ischemia pulses, which did not show any effect in control retinas, prevented the decrease in ERG a- and b-wave amplitude induced by experimental diabetes.

It was postulated that the earliest ERG manifestation of DR is a selective change in OPs. ${ }^{22,23}$ The origin of OPs has not been definitively determined, but OPs are thought to originate from feedback neural pathways in the inner retina, especially around the inner plexiform layer and mainly from amacrine cells, although ganglion cells and bipolar cells may contribute to some parts of the OPs. ${ }^{24-26}$ However, contrary to our expectations, the sum of OPs significantly decreased 9 weeks after the diabetes onset, later than changes observed in the ERG a- and b-wave amplitude. The reasons for this discrepancy are unclear, 
A
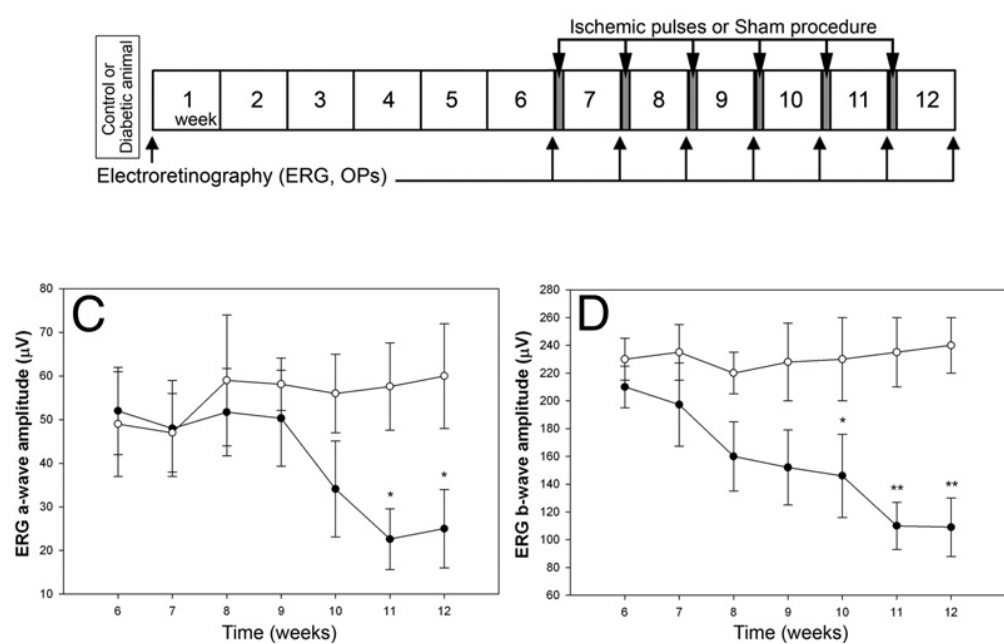
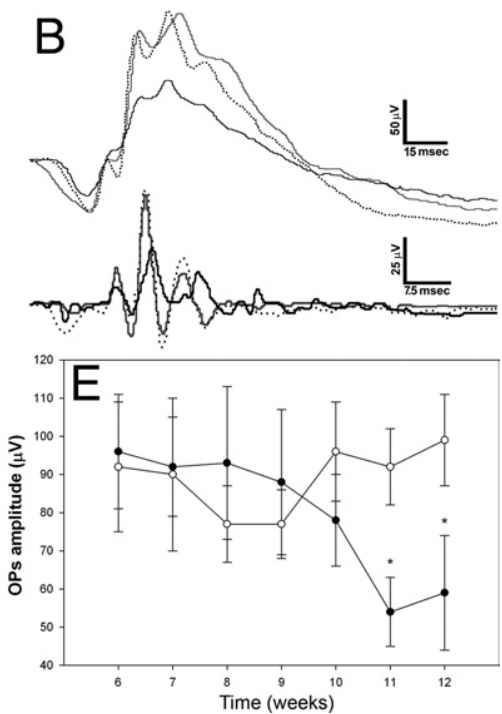

Figure 8. Temporal course of ERG protection induced by the application of brief pulses of ischemia, beginning 6 weeks after the diabetes induction A: Experimental groups and protocols for functional studies. The application of ischemia pulses started after 6 weeks of diabetes induction and continued until week 12. The ERGs (C and D) and OPs (E) were weekly assessed until week 12. After 6 weeks of diabetes, a decrease in ERG response was observed in diabetic animals (black circles), which progressed over time in sham-treated eyes. A significant protection of ERG a- and b-wave amplitude was observed after four and five ischemia pulses, respectively (white circles). Similar results were observed for OP amplitude. Note that the protection induced by ischemia persisted until week 12. B: Representative scotopic ERG and OP traces from an eye assessed 6 weeks after induction of diabetes without treatment (doted trace), an eye with a sham treatment registered 12 weeks after induction of diabetes (black trace), and an eye submitted to ischemia pulses (gray trace), which started at week 6. Values for ERG a- and b-wave and OP amplitude in nondiabetic eyes were $80.1 \pm 17.5 \mu \mathrm{V}, 289.7 \pm 25.8 \mu \mathrm{V}$, and $129.3 \pm 35.2 \mu \mathrm{V}$, respectively. These values did not change significantly until week 12 (data not shown). Data are the mean \pm SEM ( $n=10$ eyes per group); ${ }^{*} P<0.05,{ }^{* *} P<0.01$ versus sham-treated eyes, by Tukey's test.

but it may arise from a high variability of the OP data. Notwithstanding, the decrease in OP amplitude was also significantly reduced in eyes from diabetic animals exposed to ischemia pulses.

The time course of ischemic damage in the retina is similar to that observed in other regions of the central nervous system. It appears that a "maturation" phenomenon occurs whereby histologic damage becomes more evident with increasing times after ischemia and that the functional (ERG) disturbance predates morphologic changes. ${ }^{27}$ Our results further support that functional measurements revealed injury at a time when retinal morphologic features appear relatively normal because no evident changes in retinal morphologic features were observed in eyes from diabetic animals even 10 weeks after the diabetes onset. Although it is becoming increasingly clear that retinal cells are also affected by diabetes, the degeneration rate of the inner retina appears to vary in different animal models, with a clear progression over the time of diabetes. ${ }^{1}$ The present results indicate that, at least after 10 weeks of streptozotocin injection, no differences in the number of cells in the GCL and NeuN-immunopositive cells between diabetic and nondiabetic groups were observed. In agreement with this result, Barber et $\mathrm{al}^{28}$ demonstrated atrophy of the rat retina after 7.5 month of diabetes, and ganglion cell dendritic field abnormalities have been detected in human eyes with longstanding diabetes. ${ }^{29}$ Experiments are planned to address alterations in the viability of retinal cells induced by long-lasting diabetes and their eventual rescue by ischemia pulses.

Evans blue has been widely used for BRB studies in several species. ${ }^{19,30}$ Because intravenously injected Evans blue binds irreversibly to serum albumin, its distribution reflects albumin exchange between the intra- and extravascular compartments. In nondiabetic eyes without or with ischemia pulses, no leakage of Evans blue was observed, whereas in diabetic retinas submitted to a sham procedure, extravasated Evans blue was evident. Ischemia pulses prevented the effect of diabetes on this parameter.

Astrocytes are closely associated with retinal vessels, ${ }^{31}$ helping to maintain their integrity ${ }^{32}$ and increasing the barrier properties of the retinal vascular endothelium. ${ }^{33}$ Dysfunction of astrocytes has been reported to play a pivotal role in inner BRB breakdown, resulting in the production of vasogenic edema. ${ }^{34-36}$ In agreement with other reports, ${ }^{37}$ experimental diabetes induced a decrease in astrocyte GFAP immunoreactivity, ${ }^{38}$ which was restored by ischemia pulses. Barber et $\mathrm{al}^{38}$ hypothesized that a reduction in GFAP expression in retinal astrocytes during diabetes may be linked to a reduced ability to induce and maintain BRB characteristics in endothelial cells. Thus, changes in GFAP immunoreactivity could account for Evans blue leakage in diabetic eyes without and with ischemia pulses.

Although it is well known that diabetes leads to a BRB breakdown, the cellular site and mechanism involved are still controversial. On the basis of electron microscopic immunolocalization of albumin, it was suggested that BRB compromise is principally mediated by transendothelial transport of serum proteins in endocytic vesiclelike structures of approximately 0.40- to 1.0- $\mu \mathrm{m}$ diameter, whereas some additional retinal vascular leakage occurs through the interendothelial cell tight junctions, but only when adjacent vascular endothelial cells show degenerative changes. ${ }^{39}$ Moreover, using lanthanum tracing and freeze-fracture studies, ${ }^{40}$ alterations in endothelial permeability and increased number of large vesicles, later on identified as a trans-endothelial transport system of proteins such as albumin, ${ }^{39}$ were demonstrated, without 
evidences of BRB breakdown. As shown herein, frequent edemas without affecting the tight junction integrity and intraendothelial cell vesicles (approximately $1 \mu \mathrm{m}$ in diameter) filled with lanthanum were found in the retina of diabetic animals without ischemia pulses. In eyes submitted to ischemia pulses, intraendothelial cell vesicles filled with lanthanum persisted, but edemas were not observed. Thus, the absence of edemas could contribute to the decrease in extravascular albumin levels shown in diabetic eyes submitted to ischemia pulses.

The precise mechanisms responsible for the retinal protection induced by ischemia pulses remain to be established. The BRB plays an important role in the homeostatic regulation of the microenvironment in the retina. Disruption of the BRB associated with increased vascular permeability results in vasogenic edema and tissue damage, with consequent adverse effects on vision. Factors such as enhanced production of VEGF underlie the increased permeability of the BRB, and inhibition of VEGF is beneficial. ${ }^{41}$ In that context, it was shown that VEGF induces alterations of tight junction proteins, which result in BRB breakdown. Moreover, VEGF, as an angiogenesis inducer, plays a pivotal role in DR and is implicated as the initiator and mediator of nonproliferative and proliferative DRs. ${ }^{42,43}$ Repeated injections of high concentrations of VEGF in eyes of nondiabetic monkeys provoke retinal changes that resemble those observed in the early stages of DR, including vascular tortuosity and microaneurysms. ${ }^{44,45}$ Clinical trials using anti-VEGF therapies are showing promising results against advanced stages of DR. ${ }^{46,47}$ Thus, without excluding the activation of other transduction pathways associated with retinal IPC, such as adenosine, $\mathrm{K}_{\text {ATP }}$ channels, protein kinase $\mathrm{C}, 12,48-49$ and $\mathrm{NO},{ }^{50}$ among others, and based on the fact that ischemia pulses abrogated the increase in VEGF, it is tempting to speculate that the induction of ischemic conditioning could behave as an anti-VEGF therapy. In fact, it was shown that injection of VEGF into healthy eyes of animals can induce diabetes-associated ocular diseases. ${ }^{51}$ Notwithstanding, the involvement of other mechanisms, particularly stress proteins such as heat shock proteins, which have been implicated in IPC and are crucial to the survival of retinal ganglion cells, cannot be excluded. ${ }^{52}$

Both IPC and postconditioning are highly effective strategies to protect the retina from an acute and deleterious ischemic episode. These results suggest that weekly pulses of ischemia induced retinal protection against chronic ischemia, which is one of the DR hallmarks. Although the influence of duration and frequency of ischemia pulses on retinal protection deserves to be examined, these results indicate that an almost complete restoration of retinal alterations can be achieved by the procedure performed herein. However, the translational relevance of these results is limited by the fact that application of ischemia pulses started before the appearance of retinal changes provoked by diabetes. Therefore, the following experiments were performed to analyze whether ischemia pulses could not only prevent but also reduce DR progression. Human DR is a progressive optic neuropathy. In agreement, different stages of retinal dys- function were identified in experimental DR induced by streptozotocin, showing the following characteristics: i) 3 weeks of diabetes: no changes in ERG and OPs (eg, asymptomatic DR); ii) 6 weeks of diabetes: decrease in ERG b-wave without changes in the ERG a-wave and OPs (eg, moderated DR); and iii) 10 weeks of diabetes: further decrease in the ERG b-wave (versus 6 weeks) and a significant decrease in the ERG a-wave and OP amplitude (eg, advanced DR). Thus, we started the application of ischemia pulses at 6 weeks of diabetes onset, a time point in which a functional alteration is already evident. As shown herein, the delayed treatment resulted in a significant protection when compared with diabetic eyes submitted to a sham procedure, supporting that the induction of ischemic tolerance not only prevented but also restored the retinal function.

How relevant these experimental data are to human $\mathrm{DR}$ is still an open question. Although care must to be taken when extrapolating data generated in rodents to humans, rodent retinas lacking the ability to develop bona fide proliferative DR exhibit almost all of the biochemical, pathophysiologic, and histopathologic features of background retinopathy. Many exogenously delivered chemical preconditioning agents (eg, inflammatory cytokines, anesthetics, and metabolic inhibitors) can also induce ischemic tolerance, raising the hope that in the future, IPC and postconditioning could be pharmacologically mimicked in vivo. ${ }^{13}$ Therefore, the present results support that induction of ischemic tolerance could constitute a fertile avenue for the development of new therapeutic strategies in DR treatment.

\section{References}

1. Kern TS, Barber AJ: Retinal ganglion cells in diabetes. J Physiol 2008, 586:4401-4408

2. Kowluru RA, Chan PS: Oxidative stress and diabetic retinopathy. Exp Diabetes Res 2007, 2007:43603-43614

3. Zhang X, Bao S, Hambly BD, Gillies MC: Vascular endothelial growth factor-A: a multifunctional molecular player in diabetic retinopathy. Int J Biochem Cell Biol 2009, 41:2368-2371

4. Antonetti DA, Barber AJ, Bronson SK, Freeman WM, Gardner TW, Jefferson LS, Kester M, Kimball SR, Krady JK, LaNoue KF, Norbury CC, Quinn PG, Sandirasegarane L, Simpson IA; JDRF Diabetic Retinopathy Center Group: Diabetic retinopathy: seeing beyond glucose-induced microvascular disease. Diabetes 2006, 55:2401-2411

5. Yu PK, Yu DY, Alder VA, Su EN, Cringle SJ: Intracellular structures of retinal vascular endothelium in normal and early diabetic rats. Aust $N$ Z J Ophthalmol 1998, 26:S53-55

6. Wei M, Ong L, Smith MT, Ross FB, Schmid K, Hoey AJ, Burstow D, Brown L: The streptozotocin-diabetic rat as a model of the chronic complications of human diabetes. Heart Lung Circ 2003, 12:44-50

7. Kern TS: In vivo models of diabetic retinopathy. Diabetic retinopathy. Edited by EJ Duh. Totowa, NY, Humana Press, 2009, pp 137-156

8. Harhaj NS, Antonetti DA: Regulation of tight junctions and loss of barrier function in pathophysiology. Int J Biochem Cell Biol 2004 36:1206-1237

9. Aiello LP, Gardner TW, King GL, Blankenship G, Cavallerano JD, Ferris FL 3rd, Klein R: Diabetic retinopathy. Diabetes Care 1998, 21:143-156

10. Frank RN: On the pathogenesis of diabetic retinopathy: a 1990 update. Ophthalmology 1991, 98:586-593

11. Roth S, Li B, Rosenbaum PS, Gupta H, Goldstein IM, Maxwell KM, Gidday JM: Preconditioning provides complete protection against retinal ischemic injury in rats. Invest Ophthalmol Vis Sci 1998, 39: $777-785$ 
12. Roth S: Endogenous neuroprotection in the retina. Brain Res Bull 2004, 62:461-466

13. Gidday JM: Cerebral preconditioning and ischaemic tolerance. Nat Rev Neurosci 2006, 7:437-448

14. Wegener S, Gottschalk B, Jovanovic V, Knab R, Fiebach JB, Schellinger PD, Kucinski T, Jungehülsing GJ, Brunecker P, Müller B, Banasik A, Amberger N, Wernecke KD, Siebler M, Röther J, Villringer A, Weih M; MRI in Acute Stroke Study Group of the German Competence Network Stroke: Transient ischemic attacks before ischemic stroke: preconditioning the human brain? a multicenter magnetic resonance imaging study. Stroke 2004, 35:616-621

15. Na HS, Kim YI, Yoon YW, Han HC, Nahm SH, Hong SK: Ventricular premature beat-driven intermittent restoration of coronary blood flow reduces the incidence of reperfusion-induced ventricular fibrillation in a cat model of regional ischemia. Am Heart J 1996, 132:78-83

16. Zhao H, Sapolsky RM, Steinberg GK: Interrupting reperfusion as a stroke therapy: ischemic postconditioning reduces infarct size after focal ischemia in rats. J Cereb Blood Flow Metab 2006, 26:11141121

17. Pignataro G, Meller R, Inoue K, Ordonez AN, Ashley MD, Xiong Z, Gala R, Simon RP: In vivo and in vitro characterization of a nove neuroprotective strategy for stroke: ischemic postconditioning J Cereb Blood Flow Metab 2008, 28:232-241

18. Fernandez DC, Bordone MP, Chianelli MS, Rosenstein RE: Retinal neuroprotection against ischemia-reperfusion damage induced by postconditioning. Invest Ophthalmol Vis Sci 2009, 50:3922-3930

19. Ma N, Hunt NH, Madigan MC, Chan-Ling T: Correlation between enhanced vascular permeability, up-regulation of cellular adhesion molecules and monocyte adhesion to the endothelium in the retina during the development of fatal murine cerebral malaria. Am J Pathol 1996, 149:1745-1762

20. DePace DM: Distribution of intravascularly injected lanthanum ions in ganglia of the autonomic nervous system of the rat. J Auton Nerv Syst 1984, 11:339-347

21. Lowry $\mathrm{OH}$, Rosebrough NJ, Farr AL, Randall RJ: Protein measurement with the Folin Phenol reagent. J Biol Chem 1951, 193:265-275

22. Shinoda K, Rejdak R, Schuettauf F, Blatsios G, Völker M, Tanimoto N, Olcay T, Gekeler F, Lehaci C, Naskar R, Zagorski Z, Zrenner E: Early electroretinographic features of streptozotocin-induced diabetic retinopathy. Clin Experiment Ophthalmol 2007, 35:847-854

23. Hancock HA, Kraft TW: Oscillatory potential analysis and ERGs of normal and diabetic rats. Invest Ophthalmol Vis Sci 2004, 45:10021008

24. Heynen $\mathrm{H}$, Wachtmeister L, van Norren D: Origin of the oscillatory potentials in the primate retina. Vision Res 1985, 25:1365-1373

25. Wachtmeister L: Oscillatory potentials in the retina: what do they reveal. Prog Retin Eye Res 1998, 17:485-521

26. Rangaswamy NV, Zhou W, Harwerth RS, Frishman LJ: Effect of experimental glaucoma in primates on oscillatory potentials of the slowsequence mfERG. Invest Ophthalmol Vis Sci 2006, 47:753-767

27. Rosenbaum DM, Rosenbaum PS, Singh M, Gupta G, Gupta H, Li B, Roth S: Functional and morphologic comparison of two methods to produce transient retinal ischemia in the rat. J Neuroophthalmol 2001, 21:62-68

28. Barber AJ, Lieth E, Khin SA, Antonetti DA, Buchanan AG, Gardner TW: Neural apoptosis in the retina during experimental and human diabetes: early onset and effect of insulin. J Clin Invest 1998, 102: 783-791

29. Meyer-Rüsenberg B, Pavlidis M, Stupp T, Thanos S: Pathological changes in human retinal ganglion cells associated with diabetic and hypertensive retinopathy. Graefes Arch Clin Exp Ophthalmol 2007, 245:1009-1018

30. Zhang SX, Ma JX, Sima J, Chen Y, Hu MS, Ottlecz A, Lambrou GN Genetic difference in susceptibility to the blood-retina barrier breakdown in diabetes and oxygen-induced retinopathy. Am J Pathol 2005, 166:313-321

31. Schnitzer J: Astrocytes in the guinea pig, horse, and monkey retina: their occurrence coincides with the presence of blood vessels. Glia 1988,1: $74-89$
32. Zhang Y, Stone J: Role of astrocytes in the control of developing retinal vessels. Invest Ophthalmol Vis Sci 1997, 38:1653-1666

33. Gardner TW: Histamine. ZO-1 and increased blood-retinal barrie permeability in diabetic retinopathy Trans Am Ophthalmol Soc 1995 93:583-621

34. Chan-Ling T, Stone J: Degeneration of astrocytes in feline retinopathy of prematurity causes failure of the blood-retinal barrier. Invest Ophthalmol Vis Sci 1992, 33:2148-2159

35. Gardner TW, Lieth E, Khin SA, Barber AJ, Bonsall DJ, Lesher T, Rice $\mathrm{K}$, Brennan WA Jr: Astrocytes increase barrier properties and ZO-1 expression in retinal vascular endothelial cells. Invest Ophthalmol Vis Sci 1997, 38:2423-2427

36. Rungger-Brändle E, Dosso AA, Leuenberger PM: Glial reactivity, an early feature of diabetic retinopathy. Invest Ophthalmol Vis Sci 2000 , 41:1971-1980

37. Asnaghi V, Gerhardinger C, Hoehn T, Adeboje A, Lorenzi M: A role for the polyol pathway in the early neuroretinal apoptosis and glial changes induced by diabetes in the rat. Diabetes 2003, 52:506-511

38. Barber AJ, Antonetti DA, Gardner TW; The Penn State Retina Research Group: Altered expression of retinal occludin and glial fibrillary acidic protein in experimental diabetes. Invest Ophthalmol Vis Sci 2000, 41:3561-3568

39. Vinores SA, Derevjanik NL, Mahlow J, Berkowitz BA, Wilson CA: Electron microscopic evidence for the mechanism of blood-retinal barrier breakdown in diabetic rabbits: comparison with magnetic resonance imaging. Pathol Res Pract 1998, 194:497-505

40. Caldwell RB, Slapnick SM: Freeze-fracture and lanthanum studies of the retinal microvasculature in diabetic rats. Invest Ophthalmol Vis Sci 1992, 33:1610-1619

41. Kaur C, Foulds WS, Ling EA: Blood-retinal barrier in hypoxic ischaemic conditions: basic concepts, clinical features and management. Prog Retin Eye Res 2008, 27:622-647

42. Lutty GA, McLeod DS, Merges C, Diggs A, Plouét J: Localization of vascular endothelial growth factor in human retina and choroid. Arch Ophthalmol 1996, 114:971-977

43. Aiello LP, Wong JS: Role of vascular endothelial growth factor in diabetic vascular complications. Kidney Int Suppl 2000, 77:S113-S119

44. Tolentino MJ, Miller JW, Gragoudas ES, Jakobiec FA, Flynn E, Chatzistefanou K, Ferrara N, Adamis AP: Intravitreous injections of vascular endothelial growth factor produce retinal ischemia and microangiopathy in an adult primate. Ophthalmology 1996, 103:1820-1828

45. Tolentino MJ, McLeod DS, Taomoto M, Otsuji T, Adamis AP, Lutty GA: Pathologic features of vascular endothelial growth factor-induced retinopathy in the nonhuman primate. Am J Ophthalmol 2002, 133:373-385

46. Arevalo JF, Fromow-Guerra J, Quiroz-Mercado H, Sanchez JG, Wu L, Maia M, Berrocal MH, Solis-Vivanco A, Farah ME: Pan-American Collaborative Retina Study Group: primary intravitreal bevacizumab (Avastin) for diabetic macular edema: results from the Pan-American Collaborative Retina Study Group at 6-month follow-up. Ophthalmology 2007, 114:743-750

47. Ng EW, Adamis AP: Anti-VEGF aptamer (pegaptanib) therapy for ocular vascular diseases. Ann N Y Acad Sci 2006, 1082:151-171

48. Li B, Yang C, Rosenbaum DM, Roth S: Signal transduction mechanisms involved in ischemic preconditioning in the rat retina in vivo. Exp Eye Res 2000, 70:755-765

49. Sakamoto K, Kuwagata M, Nakahara T, Ishii K: Late preconditioning in rat retina: involvement of adenosine and ATP-sensitive $\mathrm{K}(+)$ channel. Eur J Pharmacol 2001, 418:89-93

50. Sakamoto K, Yonoki Y, Kubota Y, Kuwagata M, Saito M, Nakahara T, Ishii K: Inducible nitric oxide synthase inhibitors abolished histological protection by late ischemic preconditioning in rat retina. Exp Eye Res 2006, 82:512-518

51. Starita C, Patel M, Katz B, Adamis AP: Vascular endothelial growth factor and the potential therapeutic use of pegaptanib (macugen) in diabetic retinopathy. Dev Ophthalmol 2007, 39:122-148

52. Whitlock NA, Agarwal N, Ma JX, Crosson CE: Hsp27 upregulation by HIF-1 signaling offers protection against retinal ischemia in rats. Invest Ophthalmol Vis Sci 2005, 46:1092-1098 